\title{
Green material from wheat straw and starch for elevator decoration
}

\author{
Liu Junjun ${ }^{1,2, a}$, Man Zengguang ${ }^{1,2, b}$, Guo Lanzhong ${ }^{1,2, c, *}$, Dou Yan ${ }^{1,2, d}$, \\ ${ }^{1}$ Changshu Institute of Technology, Changshu, China \\ 2 Jiangsu Key Laboratory for Elevator Intelligent Safety, China \\ iamlsm01@163.com, ${ }^{\mathrm{b}} 55481981 @ q q . c o m,{ }^{\mathrm{c}} 841029689 @ q q . c o m,{ }^{\mathrm{d}} 240871949 @ q q . c o m$, \\ *corresponding author
}

Keywords: wheat straw; starch; green materials; elevator decoration

\begin{abstract}
The main goal of this work was to use wheat straw (WS) in the production of environmentally sound materials using corn based adhesives (CA). Treatments of WS with $\mathrm{NaOH}$ and hot-water were undertaken to evaluate the effect of such treatments on the performance of produced materials. The influence of material density, starch content and varieties of starch (cornstarch, cassava starch, potato starch) on properties of materials was also investigated. Results showed that cornstarch based materials had higher flexural properties. Materials made from hot-water treated straw and cornstarch had better interface and higher flexural properties, and flexural strength and flexural elastic modulus reached peak values at starch content of $10 \%$ and material density of $0.7 \mathrm{~g} / \mathrm{cm} 3$. Materials made from non-treated straw and cornstarch had lower moisture absorption. Moisture absorption was increasing with starch content increasing and density decreasing. The materials developed from this work may have potential application for ceiling panels, bulletin boards and elevator decoration material.
\end{abstract}

\section{Introduction}

Straw is rich in resources, with low density, renewable nature and lustration ${ }^{[1]}$, starch is biodegradable, renewable, with large sources and low price ${ }^{[2]}$. Crop straw and starch composites are provided with lightweight, cheap price, renewable and biodegradable characteristic, etc, and other composite materials can not match ${ }^{[3-8]}$.

Using straw and other agricultural residues for preparation of composite materials has become the focus of world. But the crushed materials (e.g. straw powder, rice husk powder, husk powder or extracted straw fiber) of agricultural residues are the main materials of the composites ${ }^{[9-11]}$, and using discarded tires, formaldehyde resins, polymers as matrix for preparation of composite ${ }^{[12-13]}$. Such composite materials have better water resistance and acoustic insulation properties, higher internal bond strength and flexibility and flexural strength, bigger fracture coefficient. But smashing 
agricultural residues and extracting fiber from straw waste lots of energy, and the matrix of the composites is difficult to degrade.

Energy saving and environmental protection are the world's urgent request. This research used wheat straw and cassava starch, corn starch, potato starch to prepare biodegradable composite materials, and focused on the influence of material density, starch content and varieties of starch on flexural strength and flexural elastic modulus, and effect of varieties of starch and straw treatments on moisture absorption.

\section{Material and Methods}

\subsection{Materials and equipments}

Cassava starch, corn starch, potato starch were food grade starch, Shandong Jincheng Food Co., Ltd. products. Wheat straw was from Liuhe, Nanjing, previous year products. $\mathrm{NaOH}$, AR (Analytical reagent); $\mathrm{Na}_{2} \mathrm{~S}_{2} \mathrm{O}_{3}, \mathrm{AR}$; borax, AR; $30 \% \mathrm{H}_{2} \mathrm{O}_{2}, \mathrm{AR} ; \mathrm{FeSO}_{4} \cdot 7 \mathrm{H}{ }_{2} \mathrm{O}, \mathrm{AR}$, the reagents above mentioned were Nanjing Chemistry reagent Ltd. products.

Pressing equipment was XLB-DC magnetic plate vulcanizing machine, Rubber Machinery Co., Ltd. Huzhou Shunli products. Performance testing machine was TMS-Pro, FTC products, US. Moisture absorption testing instrument was HPX-16085 constant temperature and moisture case, Shanghai Xinmiao Medical Treatment Ltd. products. Stereomicroscope was SMZ1000, Nikon Japan products.

\subsection{Sample preparation}

\subsubsection{Wheat straw treatments}

WS was extensively washed with distilled water in order to remove impurities (mainly dust). This operation was performed several times at room temperature and under vigorous stirring. After successive washings, WS was dried in an air-circulated oven at $103^{\circ} \mathrm{C}$. This material was stored in hermetic plastic containers in order to prevent microbial attack (i.e. fungi) before using it in followed treatments. Washed WS without any further treatments was used as control and was labeled CWS.

Some components of cellulose fibres represent a hydrophobic blockage for fibre wetting and they must be efficiently removed ${ }^{[14-16]}$. RS is rich in silica and waxes, deteriorating the properties and making WS unsuitable for textile applications ${ }^{[15,17-18]}$. In order to improve the WS wettability and performance, different treatments were applied. CWS was soaked in $2 \% \mathrm{NaOH}$ solution, for $2 \mathrm{~h}$ at room temperature with occasional shaking followed by washing with distilled water for several times to leach out the absorbed $\mathrm{NaOH}$ until neutral was reached subsequently oven dried. The $\mathrm{NaOH}$-treated CWS was labeled as NWS.

CWS was soaked in hot-water, for $2 \mathrm{~h}$ at $100^{\circ} \mathrm{C}$ followed by oven dried. The hot-water-treated CWS was labeled as HWS.

\subsubsection{Preparation of cornstarch adhesives}

Cornstarch adhesives was prepared by the dispersion of the cornstarch (CS) powder in distilled water at a CS-to-water ratio 1:10 under stirring at room temperature for $2 \mathrm{~h}$ with

$1.2 \mathrm{wt} \%$ sodium thiosulfate $\left(\mathrm{Na}_{2} \mathrm{~S}_{2} \mathrm{O}_{3} .5 \mathrm{H}_{2} \mathrm{O}\right)$ on dry basis of $\mathrm{CS}$ for preventing deterioration. The resultant adhesives were then ready to be mixed with WS. 


\subsubsection{Preparation of materials}

The materials were prepared using a hot compression molding process. Treated and untreated CWS (1 2 cm length) were blended with starch adhesives in a high-speed mixer for 10 min at room temperature. The equilibrated mixtures were subsequently hot-pressed into materials in a $10 \mathrm{~cm} \times 10$ $\mathrm{cm}$ steel mould equipped with stops to achieve the same thickness $(4.4 \mathrm{~mm})$ at certain manufacturing parameters.

\subsection{Testing method}

Flexural strength and flexural modulus were studied in accordance with the GB/T 21723-2008 "Wheat/rice-straw particleboard", loading speed was $10 \mathrm{~mm} / \mathrm{min}$. Rectangular sample: $100 \mathrm{~mm} \times 15$ $\mathrm{mm} \times 4.4 \mathrm{~mm}$. Five replications were used to calculate mean value.

Moisture absorption ratio of the materials was determined with equation:

$$
\omega=\frac{m_{t}-m_{0}}{m_{0}} \times 100 \%
$$

where: $m_{0}$-mass before moisture absorption $(\mathrm{g}), m_{t}$ - mass after moisture absorption $(\mathrm{g})$.

\section{Results and discussion}

\subsection{Comparison of flexural properties of materials}

Fig. 1 showed the flexural properties of varieties of starch based materials. From fig. 1, cornstarch based materials had higher flexural strength and flexural elastic modulus. Potato starch based materials had lower flexural strength and flexural elastic modulus. The flexural elastic modulus of potato starch based material was 18.5\% lower than flexural elastic modulus of cornstarch based materials. This could be accounted for the better stickiness, better dispersion and worse agglomeration of cornstarch. However, cassava starch and potato starch had worse stickiness and better agglomeration, and their materials had lower flexural properties.

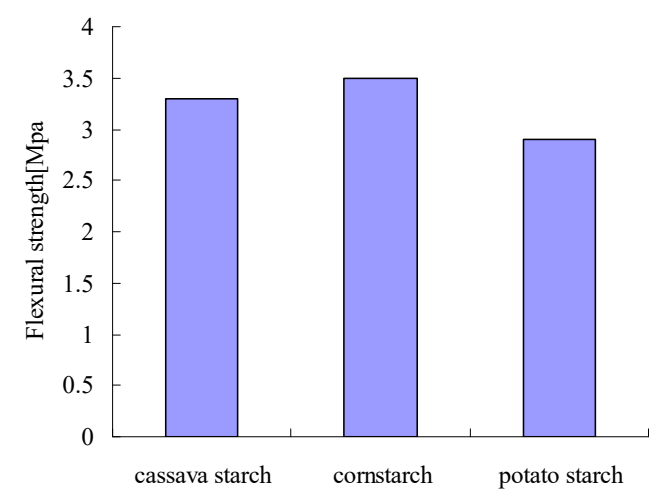

a. flexural strength

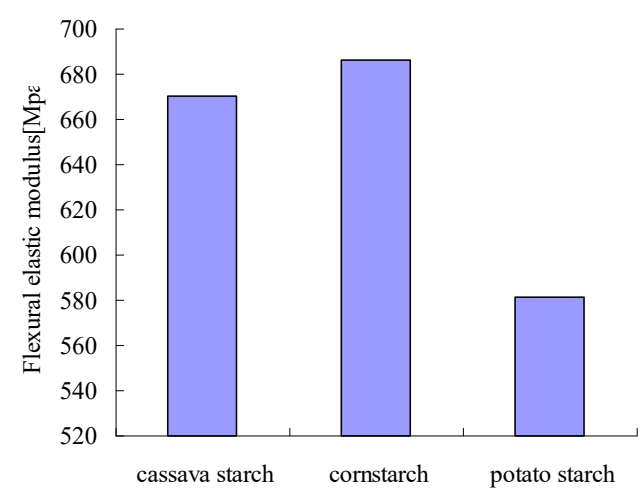

b. flexural elastic modulus

Fig. 1 Comparison of flexural properties of different starch based materials. Materials were fixed at wheat straw of $40 \mathrm{~g}$, starch content of $10 \%$ and density of $0.7 \mathrm{~g} / \mathrm{cm}^{3}$.

Fig. 2 showed the relationship between starch content and flexural properties of cornstarch based materials. From fig. 2, flexural strength and flexural elastic modulus increased and then decreased with increasing of starch content, and reached the peak value at starch mass fraction of $10 \%$. The 
flexural properties decreased with increasing of starch mass fraction above $10 \%$. This could be accounted for that the relative starch dosage was less, adhesive had weakened solidification and could not glue the wheat straw completely, so the flexural properties were lower at starch mass fraction below 10\%. Interfacial area of straw was definite, and maximum utilized quantity of starch mantled straw existed. Excrescent starch adhesive made the material internal structure looser at starch mass fraction above $10 \%$, and the flexural properties decreased.

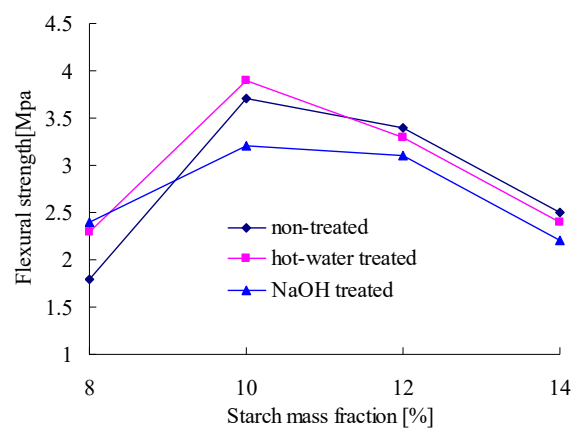

a. flexural strength

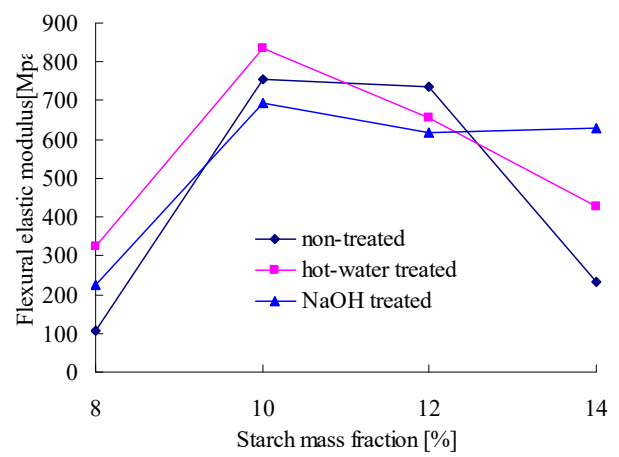

b. flexural elastic modulus

Fig. 2 Relationship between starch content and flexural properties of materials. Materials were fixed at wheat straw of $40 \mathrm{~g}$ and density of $0.7 \mathrm{~g} / \mathrm{cm}^{3}$.

Fig. 3 showed the relationship between density and flexural properties of cornstarch based materials. From fig. 3, flexural strength and flexural elastic modulus increased with increasing of material density, increased significantly with increasing of density at $0.6 \sim 0.7 \mathrm{~g} / \mathrm{cm}^{3}$, decreased slightly with increasing of density above $0.7 \mathrm{~g} / \mathrm{cm}^{3}$. This could be accounted for looser internal structure, more moisture content of materials and weakened adhesive solidification, and the flexural properties were lower at density below $0.7 \mathrm{~g} / \mathrm{cm}^{3}$. When density was $0.7 \mathrm{~g} / \mathrm{cm}^{3}$, moisture content was appropriate, starch adhesive diffuses uniformly and could glue the wheat straw completely, flexural strength and flexural elastic modulus reached the peak value. The flexural properties decreased with increasing of density above $0.7 \mathrm{~g} / \mathrm{cm}^{3}$, this was due to more compact internal structure and more difficult starch adhesive diffusion.

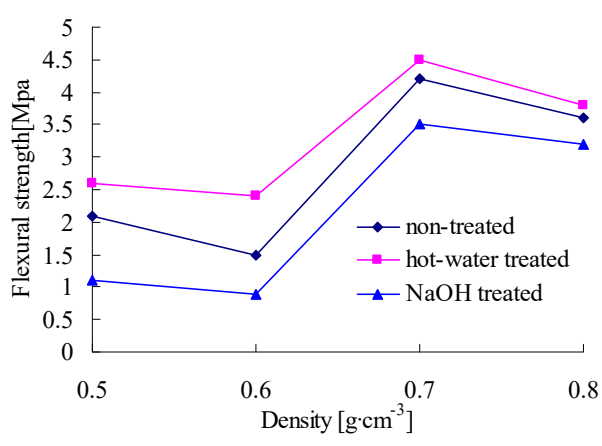

a. flexural strength

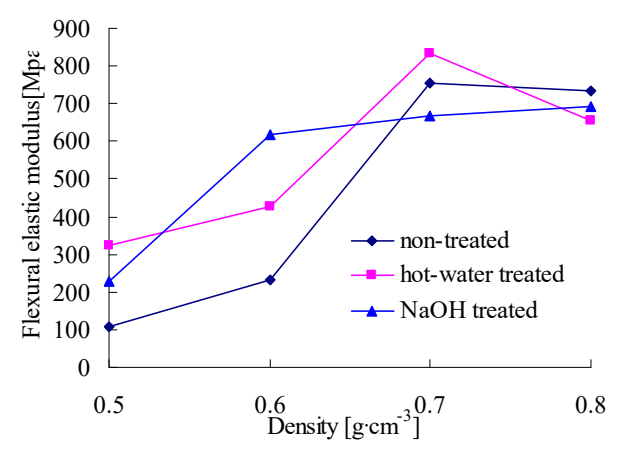

b. flexural elastic modulus

Fig. 3 Relationship between density and flexural properties. Materials were fixed at wheat straw of $40 \mathrm{~g}$ and starch mass fraction of $10 \%$.

From fig. 2 and 3, flexural properties of cornstarch based materials from hot-water treated straw were higher, and flexural properties of cornstarch based materials of $\mathrm{NaOH}$ treated straw were worst. This may be due to higher $\mathrm{SiO}_{2}$ content of straw themselves, and $\mathrm{SiO}_{2}$ forms a non-polar 
surface structure in fiber, weakening adhesive adsorption. Meanwhile, there was a wax layer on straw surface, making less friction between straw and more difficult for starch adhesive to permeate through straw, it was difficult to form "gel nails" in bonding process. All above mentioned had negative impact on materials manufacture, and decreased flexural properties of materials. Hot-water treatments could reduce $\mathrm{SiO}_{2}$ and wax content of straw effectively, making better cementation of straw and starch adhesive, and also preserving straw fibers, so materials from hot-water treated had higher flexural properties. However, $\mathrm{NaOH}$ treatments could hydrolyze straw cellulose, making straw looser and softer, lower polymerization of straw cellulose and lower flexural properties of materials from $\mathrm{NaOH}$ treated straw.

\subsection{Comparison of moisture absorption of materials}

Fig. 4a showed moisture absorption of varieties of starch based materials. From fig. 4a, moisture absorption rate of varieties of starch based materials increased with increasing of duration and obviously within 1 hour. Moisture absorption rate of cornstarch based materials increased slower. This could be accounted for that straw and starch were both hydrophilic materials that could absorb more moisture within short time. Cornstarch adhesive could stick straw better, and the surface structure was compact, therefore the moisture absorption rate was smaller in the early. Varieties of starch based materials achieved equilibrium moisture absorption rate after 18 hours. Equilibrium moisture absorption rate of cassava and potato starch based materials was $9.07 \%$ and $8.9 \%$, higher than $8.74 \%$ of cornstarch based material slightly.

Fig. $4 \mathrm{~b}$ showed moisture absorption of cornstarch based materials. From fig. 4b, moisture absorption rate of non-treated straw based materials increased slower and was smaller. This could be accounted for the existence of $\mathrm{SiO}_{2}$ and wax on straw surface, preventing water-soluble reagent penetrating through straw effectively, so non-treated straw based materials showed better water resistance.

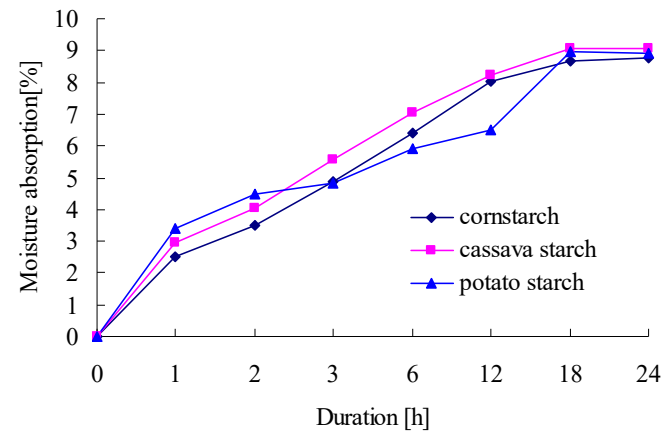

a. different starch

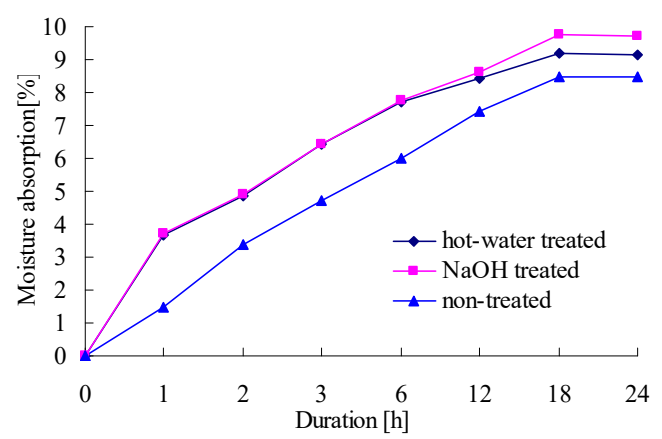

b. different treatments

Fig. 4 Comparison of moisture absorption of materials at $\mathrm{RH} 95 \%$ and $23.8^{\circ} \mathrm{C}$. Materials were fixed at wheat straw of $40 \mathrm{~g}$, starch mass fraction of $10 \%$ and density of $0.7 \mathrm{~g} / \mathrm{cm}^{3}$.

Fig. 5a showed moisture absorption of different cornstarch content materials. From fig. 5a, materials had faster increasing in prophase and higher moisture absorption rate at cornstarch mass fraction of $14 \%$, and the other three kinds materials had similar moisture absorption curves. This could be accounted for more existence of hydrophile starch on surface and in interior of materials at starch mass fraction of $14 \%$, leading to more starch content higher moisture absorption ratio and faster increasing in prophase.

Fig. $5 \mathrm{~b}$ showed moisture absorption of different density cornstarch based materials at starch mass fraction of $10 \%$. From fig. $5 \mathrm{~b}$, moisture absorption rate of lower density $\left(0.5,0.6 \mathrm{~g} / \mathrm{cm}^{3}\right)$ 
materials increased faster than others. This could be accounted for lower density less tightness of materials, leading to more moisture absorption ratio at same phase. Moreover, materials had similar equilibrium moisture absorption rate due to same weight of straw and starch.

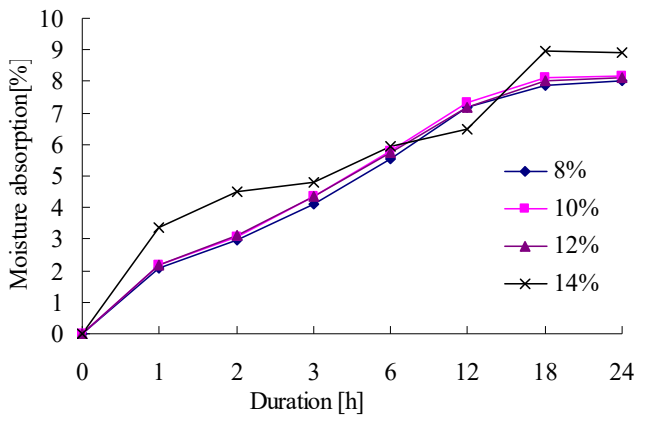

a. different cornstarch content materials

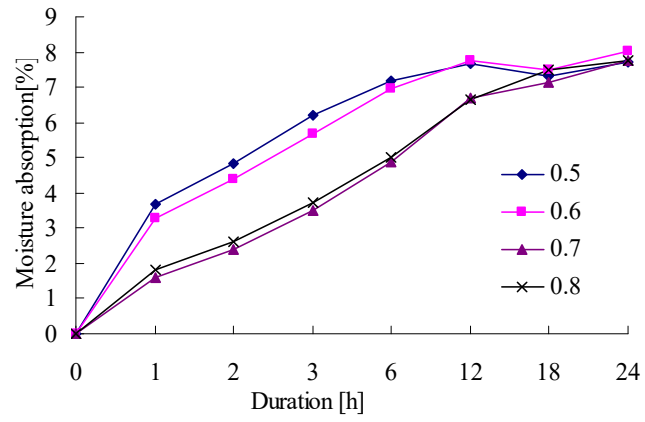

b. different density of cornstarch materials

Fig.5 Comparison of moisture absorption of materials at RH $95 \%$ and $23.8^{\circ} \mathrm{C}$. Materials were fixed at hot-water treated straw of $40 \mathrm{~g}$.

\section{Conclusions}

Cornstarch based materials had higher flexural properties. Materials from hot-water treated straw and cornstarch had better interface and higher flexural properties, and flexural strength and flexural elastic modulus reached peak values at starch mass fraction of $10 \%$ and density of $0.7 \mathrm{~g} / \mathrm{cm}^{3}$.

Materials from non-treated straw and cornstarch had lesser moisture absorption ratio, and equilibrium moisture absorption rate was $8.48 \%$. Moisture absorption ratio increased with starch content increasing and density of materials decreasing.

\section{Acknowledgements}

This work was financially supported by the College Students' practical Innovation Training Program, and Jiangsu science and technology project fund (BY2016050-04).

\section{References}

[1] Tong Jin, Ma Yunhai, Ren Luquan. Naturally biological materials and their tribology: a review [J]. Tribology, 2010, 21(4):315-320

[2] CHEN Xiaolei, SHI Jiangao, WANG Lei. Degradability of Poly(lactic-acid)/Starch Composite in Seawater [J]. Marine Fisheries, 2013, (31)4:420-425 (in Chinese)

[3] Reddy, N, Yang, Y. Preparation and characterization of long natural cellulose fibers from wheat straw [J]. J Agric Food Chem, 2007, 55:8570-8575

[4] ZHOU Dingguo, ZHANG Yang. The Development of Straw-based Composites Industry in China [J].CHINA WOOD INDUSTRY, 2007, (21)1:5-8 (in Chinese)

[5] Schirp A, Loge F, Englund K. Pilot-scale production and material properties of extruded straw-plastic composites based on untreated and fungal-treated wheat straw [J]. For Prod J, 2012b,56:90-96

[6] Alemdar A, Sain M. Biocomposites from wheat straw nanofibers:morphology, thermal and mechanical properties [J]. Compos Sci Technol, 2008,68:557-565

[7] Li X, Wang S, Duan L. Particulate and trace gas emissions from open burning of wheat straw and corn stover in China [J]. Environ Sci Technol , 2007,41:6052-6058

[8] Panthapulakkal S, Sain M. Injection molded wheat straw and corn stem filled polypropylene composites [J]. J Polym Environ, 2006, 14:265-272 
[9] Han Seung Yang, Dae Jun Kim, Young Kyu Lee. Possibility of using waste tire composites reinforced with rice straw as construction materials [J]. Bioresource Technology , 2014, 95: 61-65

[10] Soren Halvarsson, Hakan Edlund, Magnus Norgren. Properties of medium-density fibreboard (MDF) based on wheat straw and melamine modified urea formaldehyde (UMF) resin [J]. Industrial Crops and Products , 2008,28:37-46

[11] Xuan Kuang, Rui Kuang, Xiaodong Zheng. Mechanical properties and size stability of wheat straw and recycled LDPE composites coupled by waterborne coupling agents [J]. Carbohydrate Polymers, 2010, 80:927-933

[12] Andrzej K Bledzki, Abdullah A Mamun, Jürgen Volk. Physical, chemical and surface properties of wheat husk, rye husk and soft wood and their polypropylene composites [J]. Composites: Part A, 2010, 41:480-488

[13] Yi Zou, Shah Huda, Yiqi Yang. Lightweight composites from long wheat straw and polypropylene web [J]. Bioresource Technology, 2010, 101:2026-2033

[14] Leiva P, Ciannamea E M, Ruseckaite R A, Stefani P M. Medium-density particleboards from rice husks and soybean protein concentrate [J]. J Appl Polym Sci, 2013, 106: 1301-1306

[15] Ndazi B S, Karlsson S, Tesha J V, Nyanumwa C W. Chemical and physical modifications of rice husks for use as composite panels [J]. Compos Part A, 2007, 38: 925-935

[16] Wang B, Sain M, Oksman K. Study of structural morphology of hemp fiber from the micro to the nanoscale [J]. Appl Compos Mater, 2013, 14: 89-103

[17] Salam A, Reddy N, Yang Y. Bleaching of kenaf and cornhusk fibers [J]. Ind Eng Chem Res, 2007, 46: $1452-$ 1458

[18] Wójciak A, Kasprzyk H, Khmelinskii I, Krawczyk A, Oliveira A S, Ferreira L F V, Weselucha-Birczynska A, Sirorski M. Direct Characterisation of hydrogen peroxide thermomechanical pulp using spectroscopic methods [J]. J Phys Chem A, 2012, 111: 10530-10536 